\title{
Indexing Powder Patterns in Physical Form Screening: Instrumentation and Data Quality
}

\author{
ALASTAIR J. FLORENCE, ${ }^{1}$ BRUNO BAUMGARTNER, ${ }^{2}$ CHRIS WESTON, ${ }^{3}$ NORMAN SHANKLAND, ${ }^{1,4}$ \\ ALAN R. KENNEDY, ${ }^{5}$ KENNETH SHANKLAND, ${ }^{6}$ WILLIAM I. F. DAVID $^{6}$ \\ ${ }^{1}$ Department of Pharmaceutical Sciences, University of Strathclyde, Glasgow G4 ONR, UK \\ ${ }^{2}$ Stoe \& Cie GmbH, Postfach 101302, D-64213 Darmstadt, Germany \\ ${ }^{3}$ Bruker-AXS Limited, Meadowside, Mountbatten Way, Congelton, Cheshire, CW12 1DN, UK \\ ${ }^{4}$ CrystallografX Limited, 38 Queen Street, Glasgow G1 3DX, UK \\ ${ }^{5}$ Department of Pure and Applied Chemistry, University of Strathclyde, Glasgow G1 1XL, UK \\ ${ }^{6}$ ISIS Facility, Rutherford Appleton Laboratory, Chilton, Oxon OX11 0QX, UK
}

Received 29 January 2002; revised 24 February 2003; accepted 4 March 2003

\begin{abstract}
Two multisample laboratory powder diffractometers have been evaluated for the purpose of pattern indexing in the context of physical form screening. Both diffractometers utilise foil transmission geometry, primary monochromated radiation, and a position-sensitive detector. Data collected from six compounds (sotalol hydrochloride, hydroflumethiazide, verapamil hydrochloride, captopril, clomipramine hydrochloride, and famotidine) showed good angular resolution ( $F W H M$ as small as ca. $0.06^{\circ}$ ) and lattice parameters were easily obtained using the indexing program DICVOL-91. The extent of preferred orientation in each pattern was estimated using the DASH implementation of the March-Dollase function and is most evident with clomipramine hydrochloride and famotidine. Otherwise, the data compare favorably with reference capillary data sets. In conclusion, where there is a requirement to analyze 20-30 samples per day, with an emphasis on obtaining the high-quality data that are important in pattern recognition and imperative in indexing, the combination of foil transmission geometry, primary monochromated radiation, plus a position-sensitive detector is highly effective. The data also afford opportunities for crystal structure determination.

(C) 2003 Wiley-Liss, Inc. and the American Pharmacists Association J Pharm Sci 92:1930-1938, 2003 Keywords: high-throughput technologies; physical form screen; physical characterization; crystallography; X-ray powder diffractometry; powder pattern indexing; crystal structure; DICVOL-91; DASH
\end{abstract}

\section{INTRODUCTION}

The development of experimental methods for increasing the efficiency and throughput of all stages of drug discovery and development is an important area of current research within the

Correspondence to: Alastair J. Florence (Telephone: 44-141548-4877; Fax: 44-141-552-6443;

E-mail: alastair.florence@strath.ac.uk)

Journal of Pharmaceutical Sciences, Vol. 92, 1930-1938 (2003)

(C) 2003 Wiley-Liss, Inc. and the American Pharmacists Association pharmaceutical industry. The drive towards increased efficiency in physical form screening stems from the need to take the most favorable physical form (polymorph, solvate, and salt) to market in the shortest time possible, while ensuring that no unexpected transformations subsequently disrupt production.

Traditional physical form screening methodologies rely on systematically fingerprinting samples recrystallized under a wide range of conditions using spectroscopic, thermal and diffraction 
techniques; it is X-ray powder diffraction (XRPD) specifically that concerns us here. Typically, the initial stage of a screen is directed towards identifying different physical forms, for example on a search/match basis. ${ }^{1}$ Diffraction pattern quality is an issue and the point has already been made that as the quality of experimental XRPD patterns improves (i.e., more accurate measurement of $2 \theta$ and intensity, $I$ ), so the process of pattern recognition becomes easier. ${ }^{1}$ A logical extension to pattern recognition is to "add value" to the drug development process at this stage by additionally determining lattice parameters and ultimately solving novel crystal structures (Figure 1). Even more so than with pattern recognition, meeting these ambitions demands routine high-quality XRPD data collections as an integral part of the physical form screen.

The focus of attention in this communication is the importance of data quality for powder pattern indexing in the context of the workflow shown in Figure 1. We have investigated two "off-the-shelf" powder diffractometers, each with a multisample configuration suited to collecting high-quality patterns at a rate of up to ca. 30 per day (based on ca. 45 min data collection per sample-see Materials and Methods). In the context of physical form screening, the ideal instrumental specification balances the requirement for small sample quantities, minimal sample preparation, and minimum data acquisition time, good angular resolution, and accurate measurement of the positions of the first 20 reflections for indexing low-symmetry structures.

Both diffractometers utilize foil transmission geometry, primary monochromated radiation, and a position-sensitive detector (PSD). While reflection geometry tends to be more popular in industry, transmission geometry presents some definitive advantages for structural analysis where (as in this case) the materials are not strongly absorb-

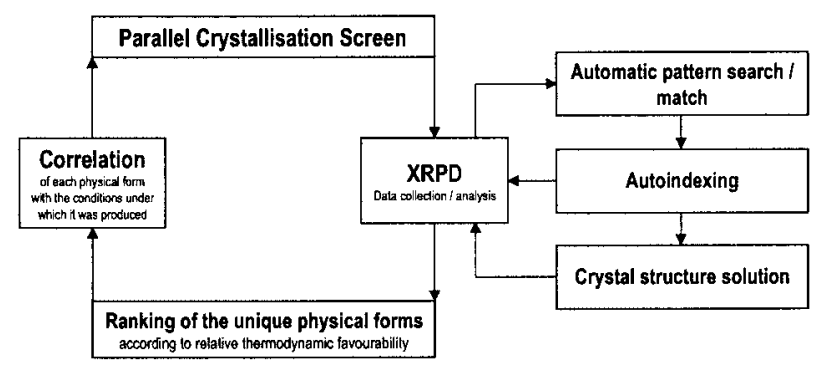

Figure 1. Flowchart illustrating the integration of $\mathrm{XRPD}$ into a physical form screen. ing. ${ }^{2}$ Similarly, while the most widely used radiation in conventional XRPD is the $\mathrm{Cu} \mathrm{K} \alpha_{1,2}$ doublet, the fact that the resolution function contains two peaks is an unnecessary complication, given the objective of accurately determining 20 reflection positions for the purpose of indexing.

In both the instruments investigated here, the convergent Guinier-type beam path using a conventional $\mathrm{X}$-ray tube line focus provides excellent resolution in the equatorial plane, but lacks resolution perpendicular to it. Therefore, a onedimensional PSD is used to maintain resolution at the expense of the complete detection of the Debye-Scherrer rings. The advantage of a linear PSD over a scintillation counter is simply a higher count rate that enables significantly faster data acquisition with very little increase in full width at half maximum ( $F W H M)$.

\section{MATERIALS AND METHODS}

Small quantities of each of six polycrystalline samples (Table 1) were analyzed using two diffractometer setups:

1. Instrument $\boldsymbol{A}$, a Bruker D8-Advance transmission diffractometer with a Johannsontype $^{3}$ curved Ge (111) focusing primary monochromator. The PSD covers an angular range of ca. $6^{\circ} 2 \theta$ simultaneously. Data were collected with each sample sandwiched between two X-ray transparent Mylar ${ }^{\mathbb{R}}$ foils using the settings listed in Table 2. The sample holder was mounted in a nine-position sample changer that allowed the sample to be rotated during data collection (Figure 2).

2. Instrument $\boldsymbol{B}$, a Stoe Stadi-P Combi transmission diffractometer with a curved Johanntype Ge (111) primary monochromator ${ }^{3,4}$ which is mounted vertically above the tube housing so that the beam path is vertical, allowing the sample stage to move within a horizontal plane while not disturbing loosely mounted specimens. The PSD covers the same angular range as above. The sample holder consists of a computer controlled $x-y$ stage into which various inserts may be clipped. For the present investigation, an X-ray transparent Kapton ${ }^{\circledR}$ foil with $12 \times 8$ sample wells ${ }^{5}$ was used (Figure 3 ) with the settings listed in Table 3. The holder was rocked in $\theta$ during data collection to improve particle statistics. 


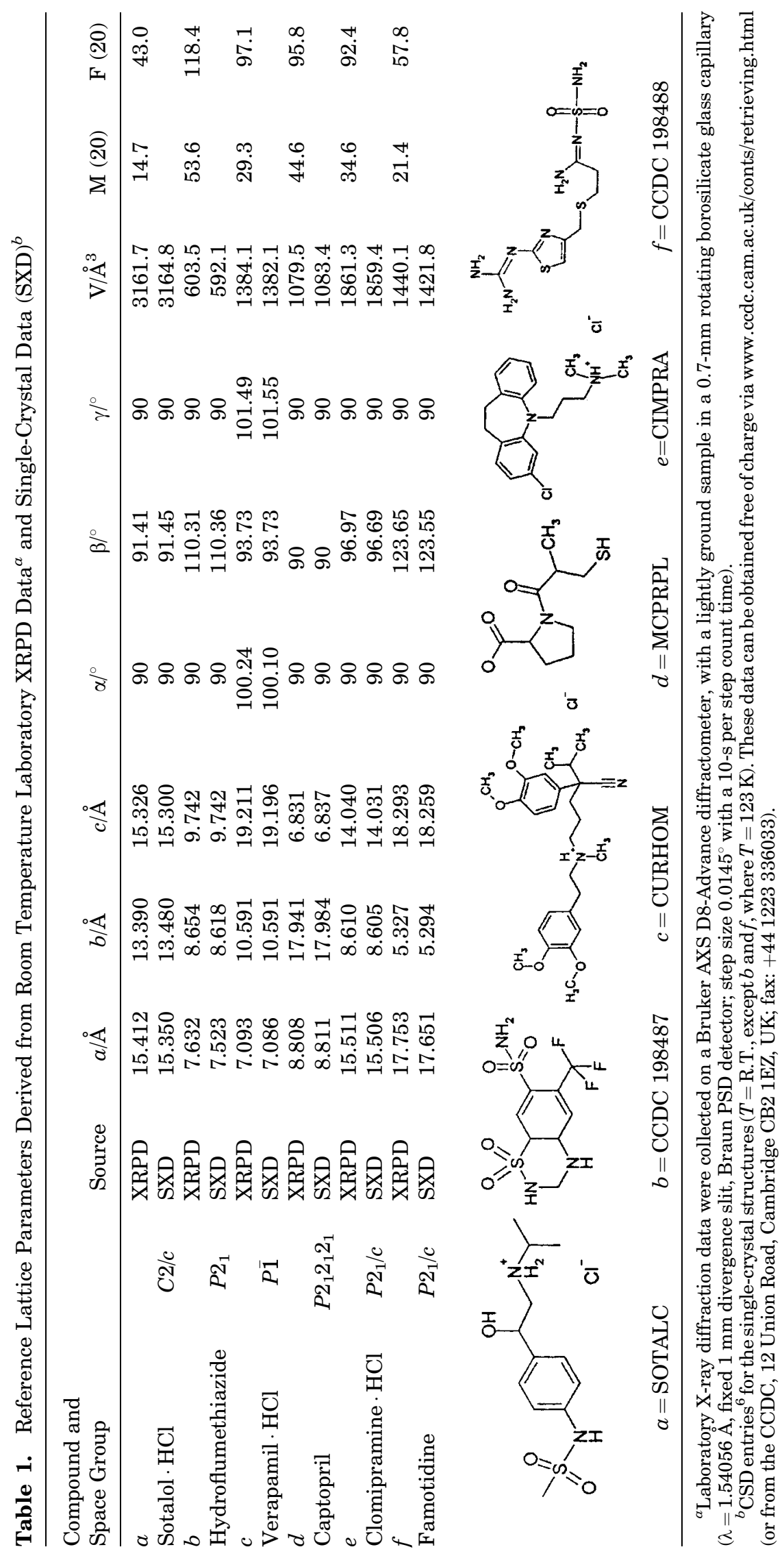


Table 2. Instrumental and Data Collection Parameters for Instrument $A$

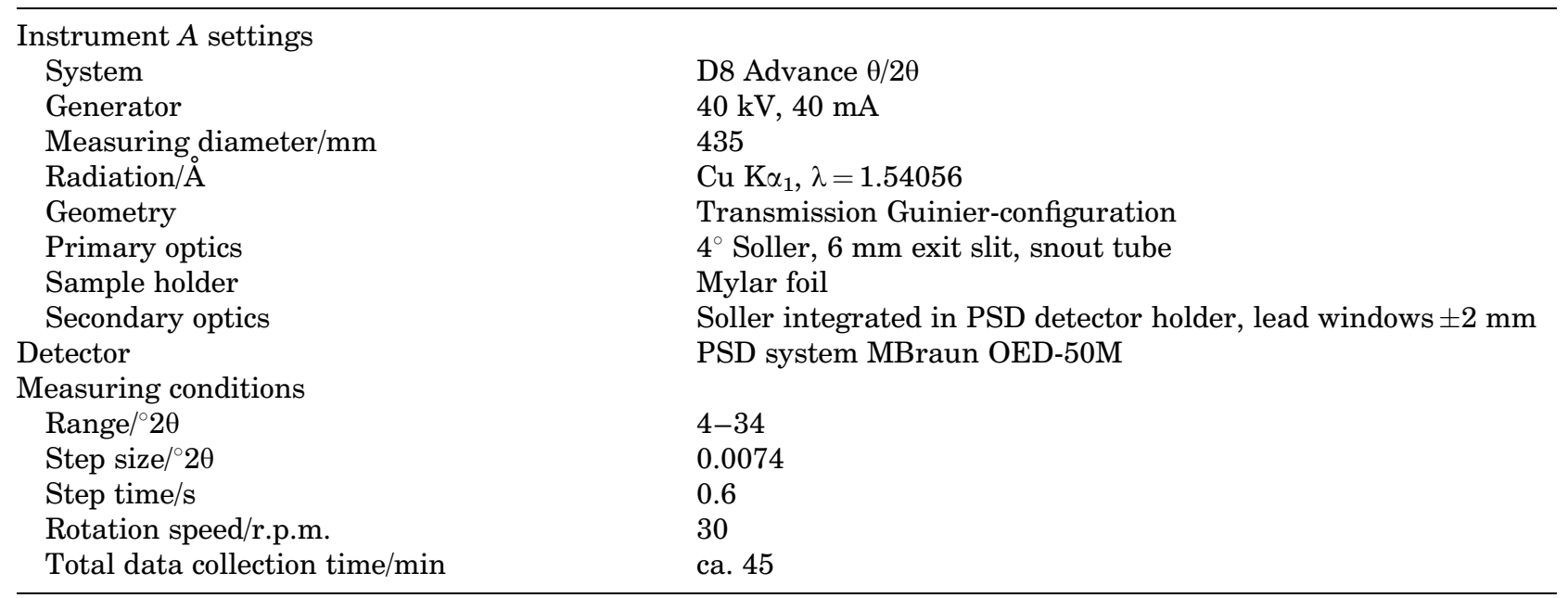

The choice of Mylar or Kapton depends on the specific application-Kapton is well suited to crystallization experiments carried out in situ because of its chemical resistance, but introduces a "hump" into the background around $5-6^{\circ} 2 \theta$ that may obscure weak reflections in that range and that is not seen with Mylar. The powder samples were mounted without grinding or other manipulations aimed at minimising particle orientation effects. In general, the potential for sample displacement error (and peak broadening) is reduced by aligning the sample carrier to the correct position for a given thickness and then minimizing thickness variations within and between samples. The six compounds were chosen because, in terms of molecular complexity, they are fairly typical of pharmaceutical active ingredients. Furthermore, a reference set of room temperature lattice parameters determined from capillary powder data were available to us, as were single-crystal structures determined at various temperatures (Table 1).

\section{RESULTS}

The XRPD powder patterns collected on instruments $A$ and $B$ from each of the six samples are shown in Figures 4 and 5, respectively. The angular resolution across each pattern is good (Table 4: mean $F W H M$ ca. $0.1^{\circ}$, min ca. $0.06^{\circ}$ ) and is comparable with the resolution obtained with the reference capillary data sets in Table 1 . The
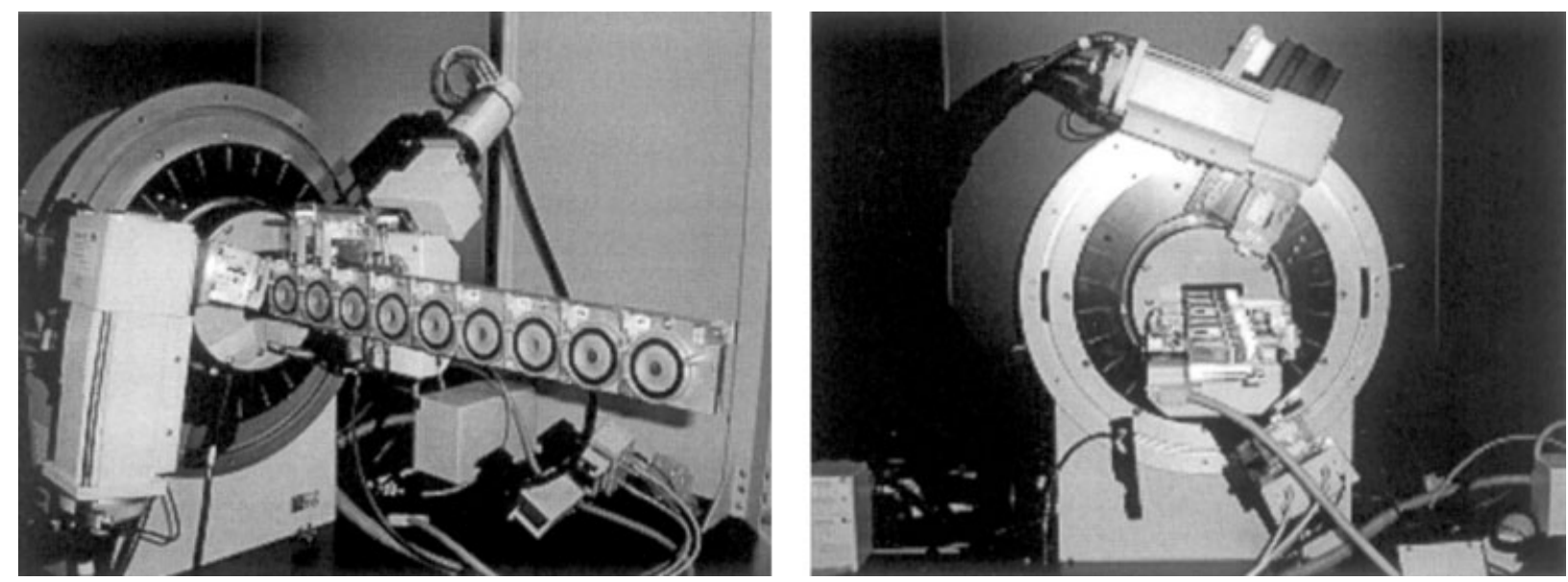

Figure 2. Instrument $A$. Data were collected with the nine-position sample changer in the configuration shown on the left, but the instrument can be easily reconfigured to have the sample in a horizontal position (right). 

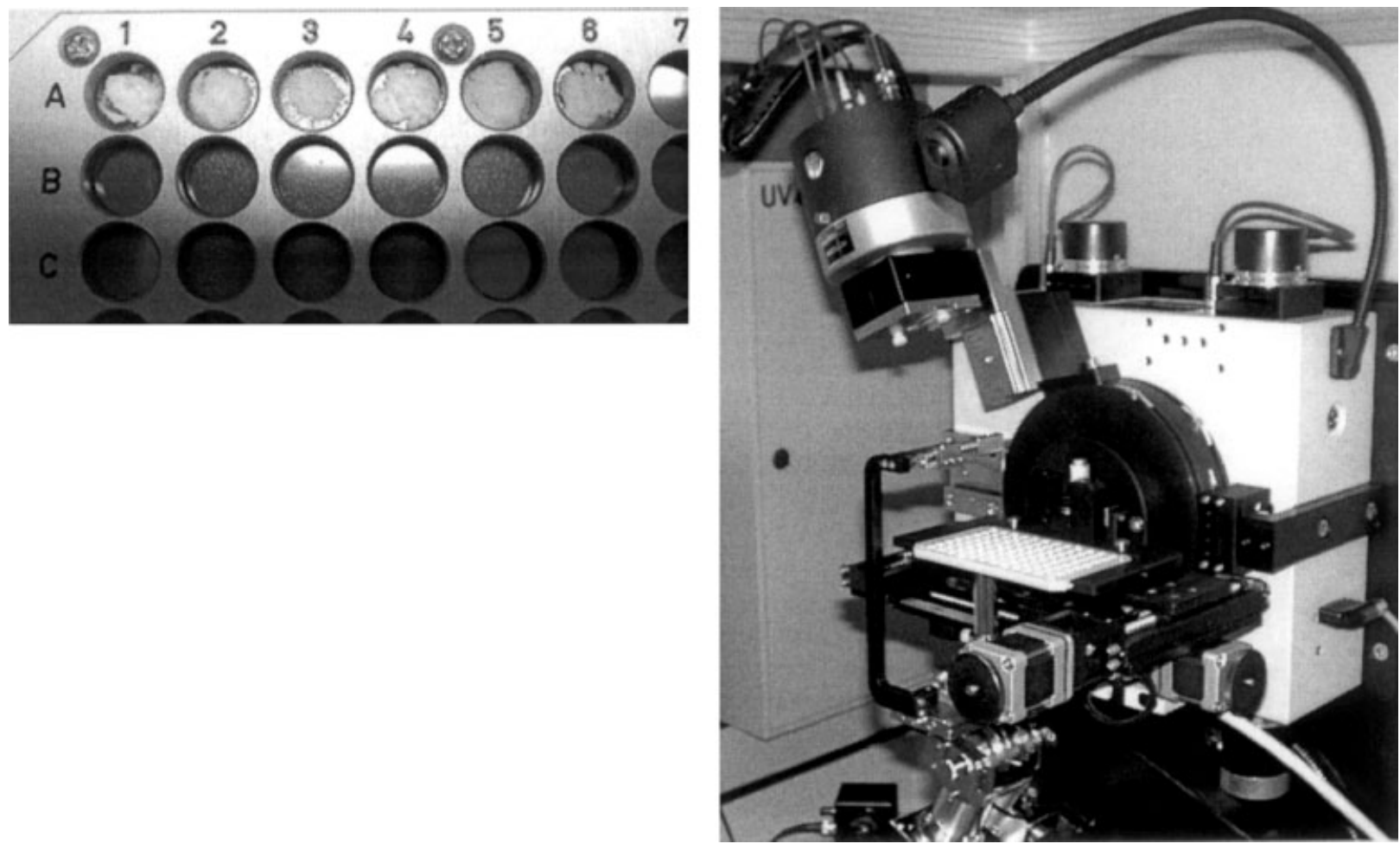

Figure 3. Instrument $B$. Samples $a$ to $f$ supported by Kapton foil in wells A1-A6 of the $12 \times 8$ sample holder (left). The $x-y$ scanning of the sample array is not limited to the sample holder shown; any coordinates within a grid of ca. $110 \mathrm{~mm}$ length $\times 65 \mathrm{~mm}$ width can be scanned in any order (right).

position of each of the first 20 peaks in each pattern was determined using $\mathrm{DASH}^{7}$ and input into DICVOL- $91^{8}$ for indexing. The output lattice parameters are listed in Table 4 and show excellent agreement with the parameters listed in Table 1.
The extent of preferred orientation in each pattern was estimated using the DASH implementation of the March-Dollase function ${ }^{9}$ (Table 5). [The March-Dollase function is adequate for describing preferred orientation at the level of structure

Table 3. Instrumental and Data Collection Parameters for Instrument $B$

\begin{tabular}{|c|c|}
\hline \multicolumn{2}{|l|}{ Instrument $B$ settings } \\
\hline System & StadiP Combi \\
\hline Generator & $50 \mathrm{kV}, 30 \mathrm{~mA}$ \\
\hline Measuring diameter/mm & 380 \\
\hline Radiation $/ \AA$ & $\mathrm{Cu} \mathrm{K} \alpha_{1}, \lambda=1.54056$ \\
\hline Geometry & Transmission Guinier-configuration \\
\hline Primary optics & Collimator $1 \times 6 \mathrm{~mm}$ \\
\hline Sample holder & $12 \times 8$ wells with Kapton foil support \\
\hline Secondary optics & Soller slit integrated in PSD holder \\
\hline Detector & Linear Stoe PSD \\
\hline \multicolumn{2}{|l|}{ Measuring conditions } \\
\hline Range/ ${ }^{\circ} 2 \theta$ & $2-39.99$ \\
\hline Step size $/{ }^{\circ} 2 \theta$ & 0.01 \\
\hline Step time/s & 0.7 \\
\hline Rocking speed $/{ }^{\circ} \theta \min { }^{-1}$ & 10 \\
\hline Total data collection time/min & ca. 45 \\
\hline
\end{tabular}




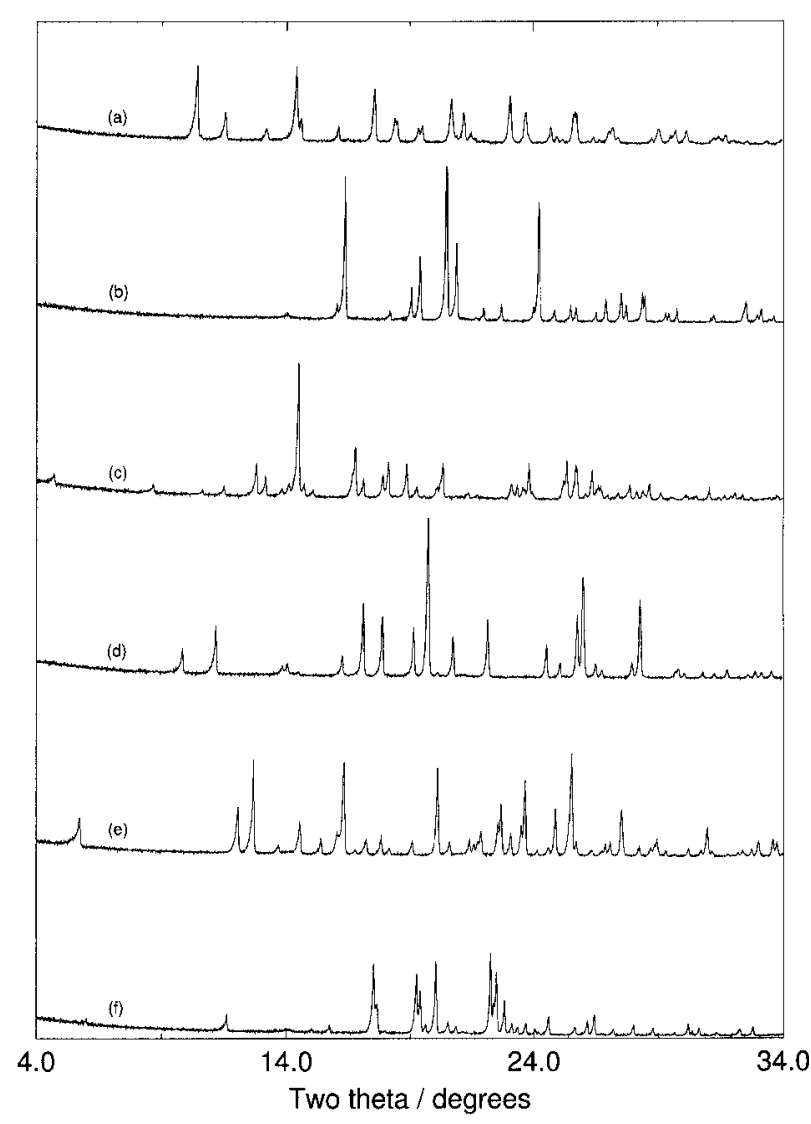

Figure 4. Instrument $A$. XRPD patterns in the range $4-34^{\circ} 2 \theta$ for compounds $a-f$ of Table 1 .

determination empolyed here-for subsequent Rietveld refinement, more advanced models (such as those based on spherical harmonics) may be preferrable.] With compound $a$ on instrument $A$, for example, data were Pawley-fitted to ca. $34^{\circ} 2 \theta$, refining background, zero-point, unit cell, and peak shape parameters. Subsequent DASH optimisation of molecular orientation gave a profile $\chi^{2}=8.2$ as the best of three repeat runs using an internal coordinate description derived from the singlecrystal structure SOTALC (Table 1). (The global optimization was constrained by fixing the position and conformation of the asymmetric unit at the values observed in the single-crystal structure.) The profile $\chi^{2}$ improved to 6.4 with the inclusion of a March-Dollase preferred orientation correction in the optimization $(r=0.91)$ along direction [010] (improvements in the other directions tested, [100] and [001], were less significant).

In summary, the intensity corrections to corresponding patterns collected on instruments $A$ and $B$ (Table 5) are generally consistent with respect to

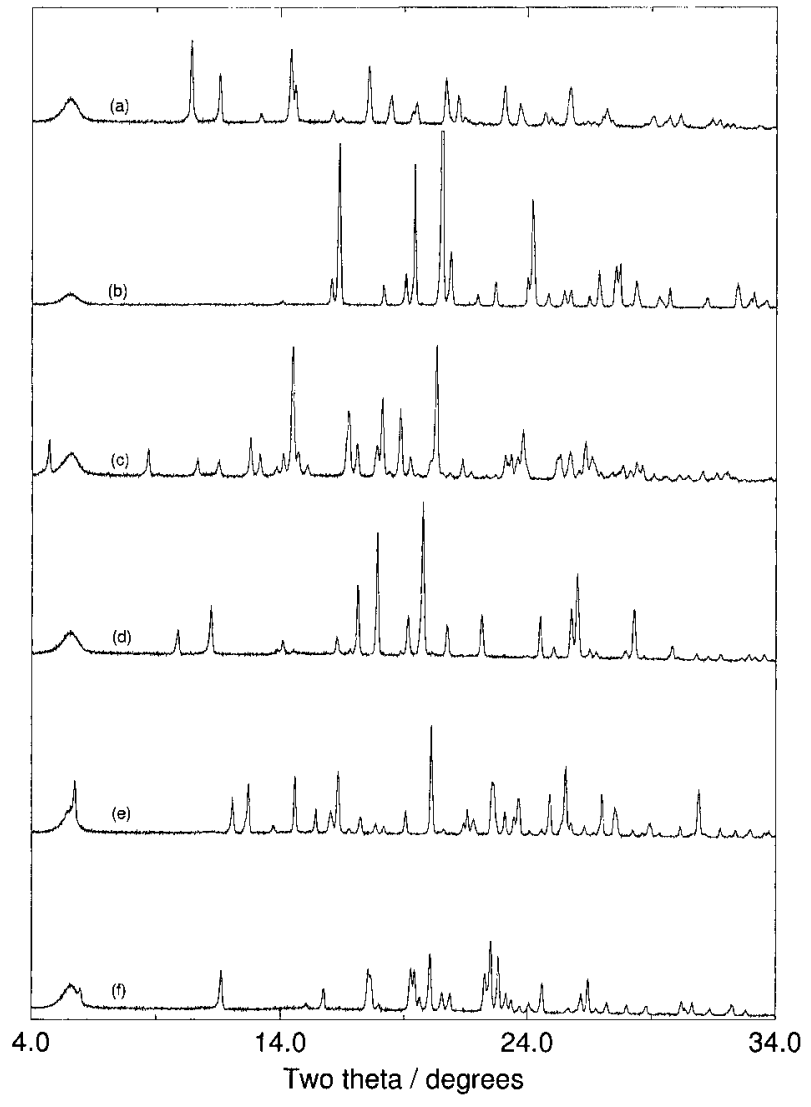

Figure 5. Instrument $B$. XRPD patterns in the range $4-34^{\circ} 2 \theta$ for compounds $a-f$ of Table 1 . The broad hump ca. $5-6^{\circ} 2 \theta$ is from the Kapton foil.

direction and overall magnitude $(r<1$ or $r>1)$ and are greatest for compounds $e$ and $f$. Among the reference capillary data sets, the effect of preferred orientation is mild for compounds $c$ and $e$ and negligible for the others.

\section{DISCUSSION}

Both instruments investigated here meet the requirements of good angular resolution and accurate reflection position measurements that are imperative for success in powder pattern indexing. In two instances (compounds $e$ and $f$ ), the effect of preferred orientation upon the transmission foil patterns is significantly greater than that observed with the capillary data, but such differences are less significant for the other compounds, where the effect of preferred orientation is relatively mild. There is some disparity between the magnitude of the corrections returned for compound $c$ on instruments $A$ and $B$, but it should 


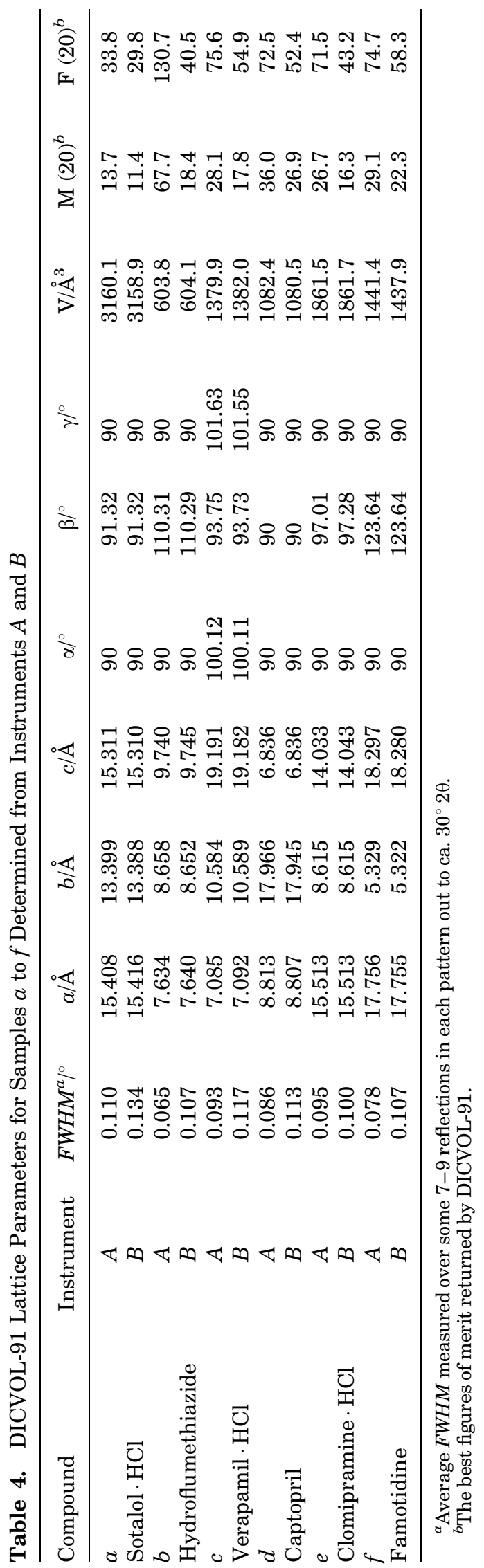

be emphasized that sample preparation for all measurements on these instruments was not optimized.

In a screening environment, suboptimal particle size and morphologies are often encountered. Variations in particle size of a given physical form will certainly have an effect on line widths. However, the resolution of the instruments described here is sufficiently good that such differences will be visible and therefore quantifiable. Crystallization of large particles or particular morphologies of a given physical form may increase the extent of preferred orientation. In such instances, greater accuracy and consistency in intensity measurments can be achieved with optimised loading of a lightly ground or sieved sample.

In summary, foil transmission data can yield a set of accurate reflection intensities where the effect of sample preferred orientation on the pattern is mild, and this creates obvious opportunities for crystal structure solution. The patterns in Figures 4 and 5 were not actually collected with structure solution in mind, and hence, the data were collected to only limited spatial resolution. Nonetheless, it is worth pointing out that even low-resolution XRPD data contains sufficient information to enable crystal structures such as hydroflumethiazide, where the total number of degrees of freedom is relatively small, to be solved by global optimisation (Figure 6 ).

Capillary remains the geometry of choice for accurate intensity measurements, but there is no doubt that filling a lightly ground sample into a glass capillary and then aligning it is significantly more time-consuming than the sample preparation needed for instruments $A$ and $B$. The latter option affords obvious advantages when it comes to maintaining the necessary throughput required to accommodate the large number of samples output from a parallel crystallization screen.

\section{CONCLUSIONS}

Where there is a requirement to analyze 20-30 polycrystalline samples per day, with an emphasis on collecting high-quality patterns (accurate measurement of $2 \theta$ and $I$ ), the combination of foil transmission geometry, primary monochromated radiation plus a PSD is highly effective. Sample throughput could obviously be increased by reducing the scan range and step time for any given pattern (data out to ca. $30^{\circ} 2 \theta$ will include the first 20 peaks in the majority of pharmaceuticals) and increasing the operating power of the X-ray 
Table 5. March-Dollase Correction of Intensities for Preferred Orientation in Data Collected Using Instruments $A$ and $B$ and in the Reference Capillary Powder Data

\begin{tabular}{|c|c|c|c|c|c|c|c|c|c|c|c|c|}
\hline \multirow[b]{2}{*}{ Compound } & \multicolumn{4}{|c|}{ Instrument $A$} & \multicolumn{4}{|c|}{ Instrument $B$} & \multicolumn{4}{|c|}{ Reference Capillary Data } \\
\hline & $\chi^{2 a}$ & $\chi^{2} \mathrm{PO}^{b}$ & Dir. & $r$ & $\chi^{2}$ & $\chi^{2} \mathrm{PO}$ & Dir. & $r$ & $\chi^{2}$ & $\chi^{2} \mathrm{PO}$ & Dir. & $r$ \\
\hline $\begin{array}{l}a \\
\text { Sotalol } \cdot \mathrm{HCl}\end{array}$ & 8.2 & 6.4 & [010] & 0.91 & 4.7 & 4.6 & [010] & 0.98 & 35.5 & 34.2 & [100] & 0.98 \\
\hline $\begin{array}{l}b \\
\text { Hydroflumethiazide }\end{array}$ & 9.0 & 3.4 & [010] & 0.90 & 45.9 & 40.9 & [010] & 0.91 & 17.3 & 13.6 & [010] & 0.97 \\
\hline $\begin{array}{l}c \\
\text { Verapamil } \cdot \mathrm{HCl}\end{array}$ & 13.1 & 5.6 & [100] & 0.78 & 12.3 & 12.3 & {$[100]$} & 0.99 & 30.9 & 19.6 & [100] & 0.92 \\
\hline $\begin{array}{l}d \\
\text { Captopril }\end{array}$ & 6.4 & 5.4 & [001] & 0.94 & 12.0 & 12.0 & {$[001]$} & 0.99 & 22.6 & 20.5 & [001] & 0.96 \\
\hline $\begin{array}{l}e \\
\text { Clomipramine } \cdot \mathrm{HCl}\end{array}$ & 37.9 & 7.4 & {$[100]$} & 1.39 & 27.6 & 21.1 & {$[100]$} & 1.18 & 30.9 & 20.8 & [100] & 1.09 \\
\hline $\begin{array}{l}f \\
\text { Famotidine }\end{array}$ & 35.8 & 7.9 & [100] & 1.66 & 6.1 & 4.4 & {$[100]$} & 1.13 & 13.8 & 12.1 & [010] & 1.04 \\
\hline
\end{tabular}

${ }^{a}$ Profile $\chi^{2}$, as defined in Ref. 7:

$$
\chi_{\text {profile }}^{2}=\frac{\sum_{i=1}^{N} w_{i}\left(y_{i}(\text { obs })-y_{i}(\text { calc })\right)^{2}}{(N-P+C)}
$$

where $y_{i}(\mathrm{obs})$ is the observed intensity at the $i$ th step in the powder diffraction pattern, $y_{i}(\mathrm{calc})$ is the associated calculated intensity; $w_{i}=1 / \sigma_{i}^{2}$, where $\sigma_{i}$, is the standard deviation of the observed intensity at that point. The summation is performed over all $N$ data points; $(N-P+C)=$ (number of data points) - (number of parameters) + (number of parameter constraints).

${ }^{b}$ Profile $\chi^{2}$ after correction of intensities for preferred orientation.

source, thereby increasing the diffracted intensity count rate, for example, $50 \mathrm{kV} / 40 \mathrm{~mA}$, or through the use of a rotating anode. Further gains could be realised by increasing the angular range of $2 \theta$, which can be scanned simultaneously by the PSD. In principle, coupling two linear PSDs would halve data collection times at no cost to the signal-tonoise, angular resolution, or the positional accu-

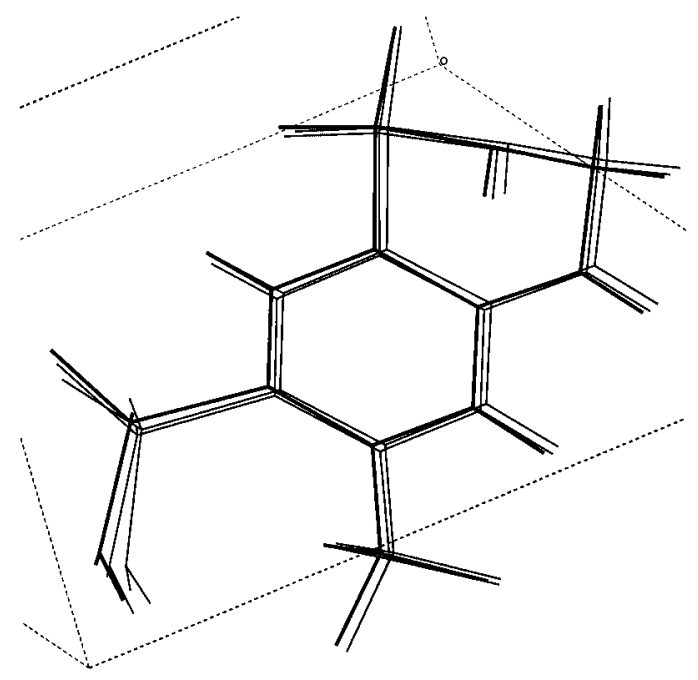

racy of observed reflections typified by Figures 4 and 5 .

Finally, the "ultimate efficiency gain" in physical form screening would see the integration of the crystallization and XRPD components of Figure 1 so as to minimize the degree of manual intervention in the process of going from powder to crystal structure. The importance of data quality to achieving this goal cannot be over emphasized.

Figure 6. The single-crystal structure of hydroflumethiazide (bold) overlaid with the DASH structures solved from 42 reflections (spatial resolution $=2.65 \AA$, Figures $4 \mathrm{~b}$ and $5 \mathrm{~b}$ ) by optimizing molecular position, orientation, and conformation. In each case, 10 repeat DASH runs converged to substantially the same crystal structure and the ratio $\chi_{\text {Rietveld }}^{2} / \chi_{\text {Pawley }}^{2}$ was sufficiently small (ca. 2) to confirm that the solution was correct. The only notable discrepancy between the single-crystal and XRPD solutions relates to the orientation of the$\mathrm{SO}_{2} \mathrm{NH}_{2}$ group. As has been noted previously, ${ }^{10}$ the $\mathrm{X}$-ray scattering power of " $\mathrm{NH}_{2}$ " is on a par with that of an O-atom, such that different orientations are essentially indistinguishable with relatively low-resolution XRPD data. 


\section{REFERENCES}

1. Faber J, Fawcett T. 2002. The Powder Diffraction File: Present and future. Acta Crystallogr B58: 325-332.

2. Louër D. 2002. Laboratory x-ray powder diffraction in structure determination from powder diffraction data. In: David WIF, Shankland K, McCusker LB, Baerlocher Ch, editors. Structure determination from powder diffraction data. IUCr monographs on crystallography, vol. 13. Oxford: Oxford University Press, pp 29-48.

3. Klug HP, Alexander LE. 1974. Crystal monochromators. In: X-ray diffraction procedures for polycrystalline and amorphous materials. New York: Wiley Interscience, pp 354-358.

4. Wölfel ER. 1983. A novel curved position-sensitive proportional counter for X-ray diffractometry. J Appl Crystallogr 16:341-348.

5. Studiengesellschaft Kohle. 2000. Deutsche Gebrauchsmusterschrift DE 29919861 U 1.
6. Allen FH. 2002. The Cambridge Structural Database: A quarter of a million crystal structures and rising. Acta Crystallogr B58:380-388.

7. David WIF, Shankland K, Cole J, Maginn S, Motherwell WDS, Taylor R. 2001. DASH user manual. Cambridge: Cambridge Crystallographic Data Centre.

8. Boultif A, Louër D. 1991. Indexing of powder diffraction patterns for low-symmetry lattices by the successive dichotomy method. J Appl Crystallogr 24:987-993.

9. Dollase WA. 1986. Correction of intensities for preferred orientation in powder diffractometry: application of the March model. J Appl Crystallogr 19: 267-272.

10. Shankland K, McBride L, David WIF, Shankland N, Steele G. 2002. Molecular, crystallographic and algorithmic factors in structure determination from powder diffraction data by simulated annealing. J Appl Crystallogr 35:443454. 